\title{
HACIA LA SOLUCIÓN DEL ENIGMA DE LA EXAGERACIÓN VERTICAL EN ESTÉREO VISIÓN
}

\section{TOWARD THE SOLUTION OF THE VERTICAL EXAGGERATION ENIGMA IN STEREO VISION}

\author{
Humberto, Rosas García \\ Geólogo y Geofísico, Asesor externo, chrosasg@yahoo.com \\ Watson Lawrence, Vargas Escobar \\ Ing. Químico, Ph.D., Postdoctoral Program, University of Pittsburgh, wlvargas@yahoo.com
}

Alexander, Ceron Correa

Ing. de Sistemas, Magíster en Ingeniería, Docente Asistente, Programa Ingeniería en Multimedia, Grupo de Investigación en Multimedia GIM, Universidad Militar Nueva Granada, Bogotá, D.C., Colombia, aceronc@umng.edu.co

Darío, Domínguez Cajeli

Matemático y Magister en Economía, Docente Investigador, Departamento de Matemáticas, Grupo de Investigación FRACUMNG, Universidad Militar Nueva Granada, Bogotá, D.C., Colombia, Fracumng@umng.edu.co

Adriana, Cárdenas Quiroga

Geóloga, Docente Tiempo Completo, Facultad de Ingeniería,

Universidad Militar Nueva Granada, Bogotá, D.C., Colombia, elsac@umng.edu.co

Fecha de recepción: Septiembre 10 de 2007

Fecha de aprobación: Octubre 30 de 2007

\section{RESUMEN}

La exageración vertical que se produce en la visión estereoscópica ha sido objeto de numerosas investigaciones, principalmente basadas en los principios de la óptica geométrica, que han dado origen a variadas teorías y formulaciones matemáticas de discutida validez científica. De allí que el problema de medir la exageración vertical estereoscópica continúe siendo un enigma. En opinión de los autores, el tema de la visión estereoscópica debe ser tratado bajo un enfoque diferente, ya no desde el punto de vista estrictamente geométrico, sino también como un fenómeno psicofísico regido por la ley de Fechner. La conexión del efecto estereoscópico con la psicofísica se evidencia por medio de experimentos basados en la observación de dibujos estereoscópicos (estereogramas). Los autores consideran que esta nueva visión del problema ofrece posibilidades para una formulación matemática confiable del fenómeno estereoscópico, y la consiguiente solución del enigma de la exageración vertical.

PALABRAS CLAVE: Estereoscopía, exageración vertical, psicofísica. 


\section{ABSTRACT}

The vertical exaggeration produced in stereoscopic vision has been the subject of numerous investigations, mainly based on optical geometry, which have given rise to several theories and mathematical formulations of questioned scientific validity. Consequently, the problem of measuring vertical exaggeration continues being an enigma. In the authors' opinion, the theme of stereoscopic vision should be approached, not only from a strict geometric point of view, but also as a psychophysical phenomenon ruled by the Fechner's law. The connection of the stereoscopic effect with psychophysics is evidenced by observing some geometric drawings under stereoscope. The authors consider that this new approach offers possibilities for a reliable mathematical formulation of the stereoscopic phenomenon, and the consequent solution of the vertical exaggeration enigma.

KEY WORDS: Stereoscopy, vertical exaggeration, psychophysics

\section{INTRODUCCIÓN}

En la visión estereoscópica la percepción de la tercera dimensión presenta todavía muchos interrogantes. Aún se desconocen sus características métricas. Lo único cierto es que las magnitudes verticales (ordenada Z) de la imagen tridimensional percibida (modelo mental), no guardan proporción con las horizontales. Por ejemplo, es bien conocido que en la observación estereoscópica de fotografías aéreas, el modelo mental suele aparecer verticalmente exagerado con relación al terreno fotografiado (modelo real). Este fenómeno comúnmente conocido como exageración vertical, ha sido objeto de numerosas investigaciones, la mayoría publicadas a comienzo de la segunda mitad del siglo pasado, sin que hasta el momento haya podido establecerse con certeza la ley física y matemática que lo rige. El tema parece haberse agotado porque no se conoce literatura reciente al respecto. En vista de tantos esfuerzos y tan escasos resultados, se ha dicho que el modelo mental, o perceptual, es un "misterio" [2], y que la búsqueda de una fórmula para determinar la exageración vertical representa un "esfuerzo quijotesco" [12].

Los autores se encuentran adelantando un Proyecto de Investigación sobre este tema. Los primeros resultados de la misma ya fueron publicados (Rosas et al, 2007), y observaciones realizadas posteriormente constituyen el tema del presente artículo.

\section{MARCO TEÓRICO}

\subsection{VISIÓN MONOCULAR}

En la visión monocular el objeto observado y la imagen retinal están conectados por relaciones geométricas según las cuales la imagen retinal es una representación exacta del objeto real en dos dimensiones, a una determinada escala. La imagen retinal es transmitida al cerebro donde se transforma en percepción o imagen mental. Debido a la perfecta co- 
nexión geométrica existente entre objeto real e imagen retinal, y la correspondencia entre ésta y la imagen mental, puede decirse que la visión monocular ofrece una representación fiel de la realidad en dos dimensiones. La situación cambia en el caso de la visión binocular o estereoscópica.

\subsection{VISIÓN ESTEREOSCÓPICA NATURAL}

En la visión estereoscópica natural, el observador percibe objetos tridimensionales a través de dos cámaras (ojos) separadas entre sí por la distancia interpupilar, de modo que el objeto tridimensional es visto desde dos ángulos. Como consecuencia, el par de imágenes retinales obtenidas, al no ser idénticas, presentan cierta disparidad denominada paralaje. Geométricamente, el paralaje permite reconstruir el objeto tridimensional observado, a partir de dos vistas planas del mismo. En geometría descriptiva es bien conocido el principio según el cual, dos vistas planas de un objeto tridimensional contienen información suficiente para reconstruir el objeto en sus tres dimensiones. Esta posibilidad de conocer y medir un objeto tridimensional a partir de dos vistas planas constituye el fundamento de la fotogrametría.

Con base en lo anterior, es claro que el cerebro dispone de los elementos geométricos necesarios para reconstruir mentalmente el objeto tridimensional observado, o modelo real, utilizando dos imágenes retinales planas, de manera similar a como lo haría un fotogrametrista. Así se obtendría un modelo mental idéntico al modelo real, con lo cual se lograría una fiel percepción de la realidad, igual a lo que ocurre en la visión monocular. Sin embargo, la experiencia muestra que el modelo mental, en vez de coincidir con el modelo real, aparece deformado en profundidad, dando lugar a lo que se ha denominado exageración vertical.

\subsection{OBSERVACIÓN DE ESTÉREO-PARES FOTOGRÁFICOS}

Además de la visión estereoscópica que se realiza por observación directa de un modelo tridimensional (visión natural), la observación puede también efectuarse utilizando un estéreo-par de fotografías. El caso más frecuente es la observación de pares de fotografías aéreas tomadas desde dos posiciones del avión, y luego vistas con ayuda de un estereoscopio. En este proceso intervienen tres modelos estereoscópicos: 1) Un modelo real representado por el área de terreno fotografiada, 2) Un modelo geométrico generado por intersección de rayos visuales cuando las fotografías son observadas a través del estereoscopio, y 3) Un modelo mental que el cerebro construye en la mente del observador, con base en la información registrada en las imágenes retinales. En la Figura 1 se muestran las relaciones entre modelo real, modelo geométrico y modelo mental cuando se observa un par de fotografías estereoscópicas.

Evidentemente, el modelo real es rígido e invariable, toda vez que representa una realidad inmodificable, al contrario de lo que ocurre con el modelo geométrico, cuyas características varían de acuerdo con las condiciones geométricas en que son observadas las fotografías. 
Básicamente, las características del modelo geométrico dependen de dos factores: 1) La geometría con que fueron tomadas las fotografías (relación $\mathrm{B} / \mathrm{H}$ ), y 2) la geometría con que éstas son observadas, dependiendo de las especificaciones ópticas del estereoscopio. En últimas, el modelo que el observador ve frente a él, es el modelo geométrico, y éste modelo genera las imágenes retinales. Por tal motivo, ha existido la tendencia a creer que las características geométricas del modelo observado (modelo geométrico), corresponden a las del modelo mental, de manera análoga a lo que ocurre en la visión monocular donde el objeto observado genera una imagen mental con igual forma geométrica. No obstante, como se mencionó anteriormente, numerosas observaciones demuestran que en la visión estereoscópica el modelo mental no coincide con el modelo geométrico, sino que presenta una exageración en su escala vertical. A diferencia de lo que ocurre en la visión monocular, en la visión estereoscópica no percibimos la realidad sino una versión distorsionada de la misma. En verdad, "nosotros no vemos el mundo exterior físico, sino sólo nuestras impresiones espaciales mentalmente proyectadas" [7].

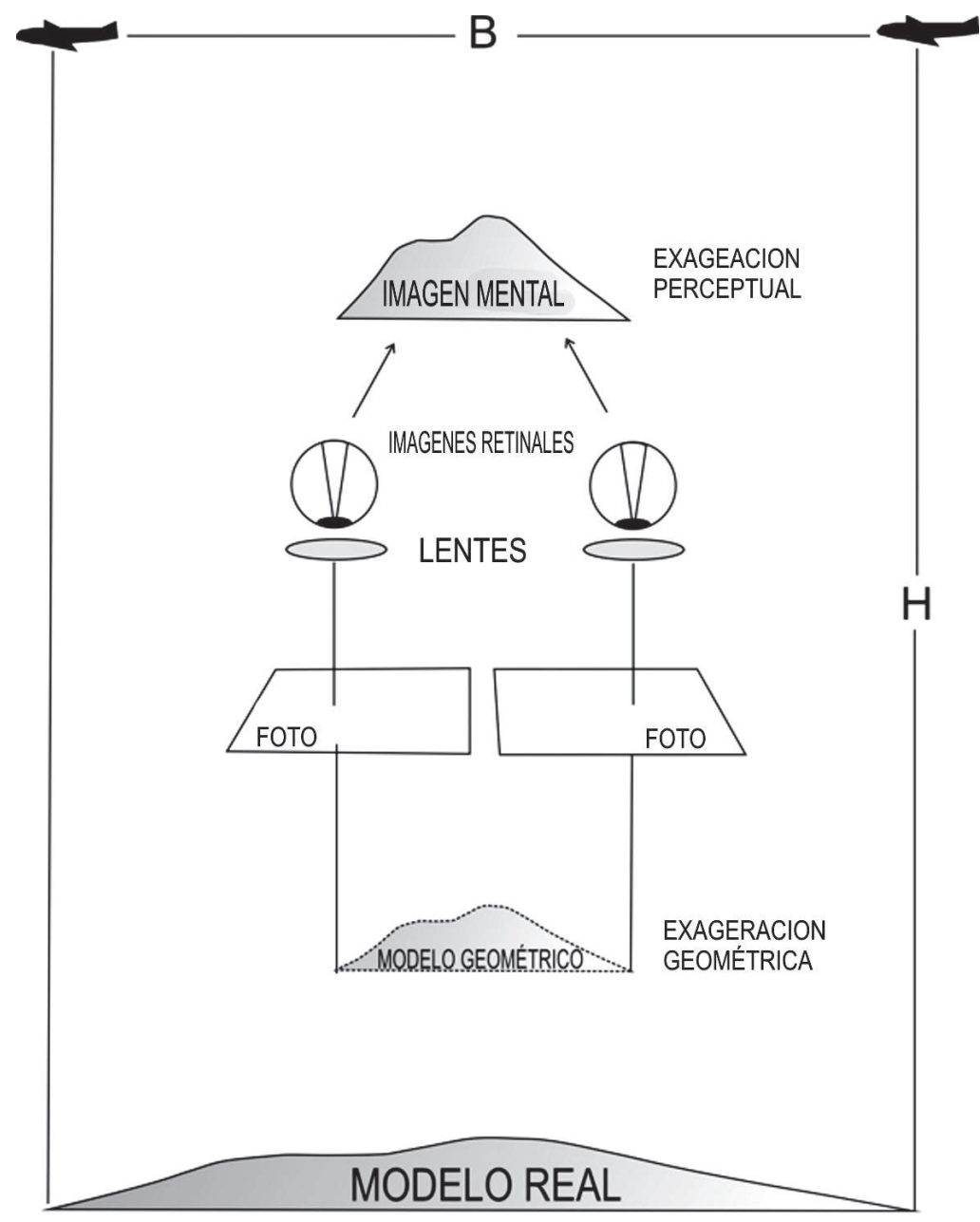

Figura 1. Observación de fotografías aéreas estereoscópicas 


\subsection{EXAGERACIÓN PERCEPTUAL Y EXAGERACIÓN GEOMÉTRICA}

La hipótesis de que el observador percibe fielmente lo que ve frente a él ha creado una confusión que aún subsiste. Si ésta hipótesis fuera cierta, el modelo mental (Figura 1) sería equivalente al modelo geométrico, y la relación entre las magnitudes verticales del modelo mental y el modelo real (exageración vertical), sería equivalente a la relación que existe entre el modelo geométrico y el modelo real. Pero los experimentos permiten comprobar que las dos relaciones no son iguales. En este aspecto, es normal encontrar actualmente en la literatura, principalmente libros de texto y manuales de manejo de instrumentos preparados por los fabricantes, cierta ambigüedad al no distinguir entre la verdadera exageración vertical estereoscópica (modelo mental /modelo real) hasta hoy desconocida, y la bien conocida relación geométrica existente entre modelo geométrico y modelo real. Esta última relación es la que suele denominarse exageración vertical. Conviene entonces aclarar que nos encontramos frente a dos conceptos de exageración vertical: 1. La exageración vertical con que el observador percibe el modelo real (modelo mental /modelo real) como resultado de un proceso mental cuya formulación matemática es desconocida, la cual podemos denominar exageración perceptual, y 2. La exageración vertical que presenta el modelo geométrico con relación al modelo real, como resultado de relaciones estrictamente geométricas, bien conocidas, y que podemos denominar exageración geométrica. Los autores utilizarán el término genérico de exageración vertical para aludir a la exageración que reviste interés por su carácter enigmático como es la exageración vertical perceptual.

\subsection{EL ENIGMA DE LA EXAGERACIÓN VERTICAL}

La determinación de la exageración vertical (perceptual) es fundamental para conocer la ley física y matemática que rige el fenómeno de la visión estereoscópica. Numerosos investigadores han tratado de resolver el problema sin mayor éxito. El tema tuvo especial auge hacia el comienzo de la segunda mitad del siglo pasado cuando aparecieron varias publicaciones, hasta que poco a poco el interés fue perdiéndose ante la falta de soluciones y enfoques nuevos. Las investigaciones se han dirigido hacia la identificación de las variables que intervienen en la visión estereoscópica, y el establecimiento de una formulación matemática que permita determinar el valor de la exageración vertical. Como resultado, se han propuesto diversas ecuaciones matemáticas, ninguna de las cuales ha demostrado ser universalmente válida. Ocho de tales ecuaciones [1], [2], [4], [5], [6], [7], [10], [12], aparecen referenciadas [8]. Recientemente no se han publicado trabajos específicos sobre este tema.

\section{NUEVO ENFOQUE DEL PROBLEMA}

Es razonable pensar que el proceso de la visión estereoscópica no es un fenómeno puramente geométrico sino también de carácter psicofísico donde intervienen, además de factores geométricos externos, ciertos elementos psíquicos internos característicos del sistema de visión humano, como se ilustra en la figura 2. El objeto tridimensional observado (modelo real) constituye el estímulo generador de una sensación visual que es el modelo 
perceptual. Por consiguiente, los autores consideran que la posibilidad de formular matemáticamente la exageración vertical se encuentra en la ley psicofísica desarrollada por G. Fechner [3] según la cual la sensación (S) es directamente proporcional al logaritmo de la intensidad del estímulo (E). O sea:

$$
S=K \log E
$$

Donde $\mathrm{K}=$ Constante de proporcionalidad.

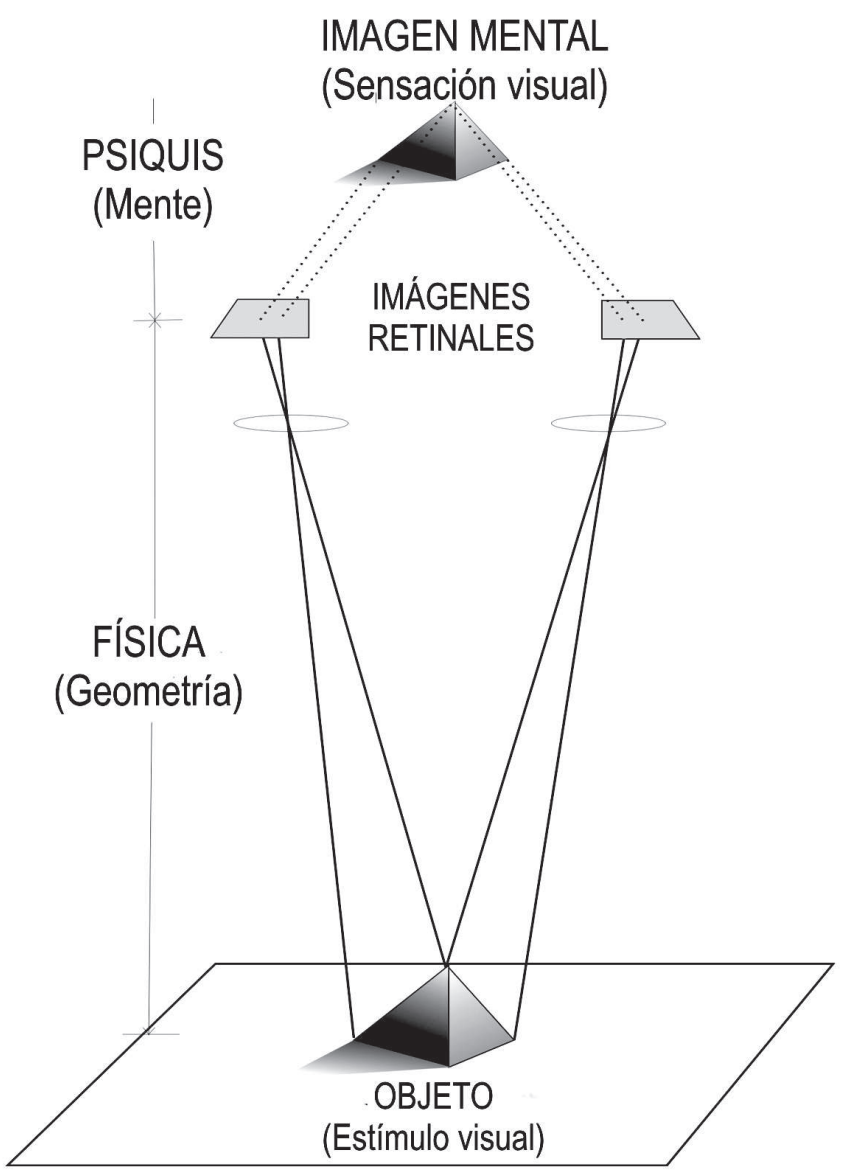

Figura 2. Proceso psicofísico de la visión estereoscópica.

\section{EVIDENCIA GRÁFICA DE UNA RELACIÓN PSICOFÍSICA}

Los autores han realizado observaciones gráficas que indican la validez de la ecuación psicofísica de Fechner [3] como ley rectora de las variables ópticas que intervienen en el proceso de la percepción estereoscópica en profundidad. Los materiales y métodos empleados se descrien a continuación. 


\section{MATERIALES Y MÉTODOS}

Los experimentos se basan en observaciones de dibujos estereoscópicos realizadas con ayuda de estereoscopio. En el presente artículo se presentan dos tipos de figuras estereoscópicas, o estereogramas (Figuras 3a y 4a), para ser observadas con estereoscopio de bolsillo. Cada una de ellas muestran el interior de una pirámide donde se perciben cinco niveles de profundidad. Considerando la posibilidad de carecer de estereoscopio para visualizar los estereogramas, se elaboraron figuras planas sombreadas con cierto efecto de perspectiva (Figuras 3b y 4b), a fin de dar una idea de lo que se observa tridimensionalmente en los estereogramas.

En el estereograma de la Figura 3a se observa el interior de una pirámide invertida, de base cuadrada, con cinco niveles de sensación de profundidad o profundidad perceptual a partir del vértice $A$. Cada uno de estos niveles perceptuales, numerados de 0 a 4 , corresponden a intervalos de paralaje de $2 \mathrm{~mm}$. Estos intervalos iguales de paralaje, que geométricamente corresponden a elevaciones iguales, no producen sensaciones iguales de profundidad, ya que a simple vista puede observarse que el espaciamiento entre los niveles perceptuales disminuye a medida que aumenta su altura con relación al vértice A. Por ejemplo, entre el nivel 3 y el 4 se aprecia una separación menor que entre el nivel 0 y 1 . Esto indica que la sensación de profundidad no varía proporcionalmente con el paralaje, o sea que, a una escala aritmética del paralaje, corresponde una escala de sensación de profundidad cuyos intervalos aumentan progresivamente en profundidad, como ocurre en una secuencia logarítimica. La comprobación de que, en efecto, las sensaciones de profundidad son proporcionales al logaritmo de los paralajes, se realizó por medio de un segundo experimento que se describe a continuación.

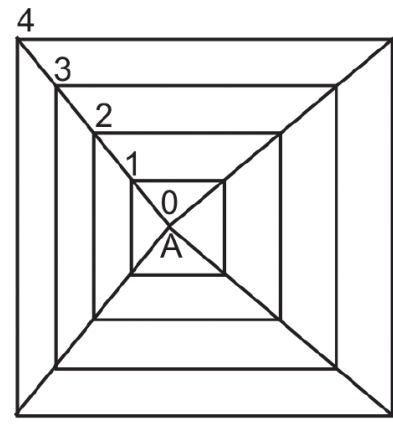

3a Estereograma

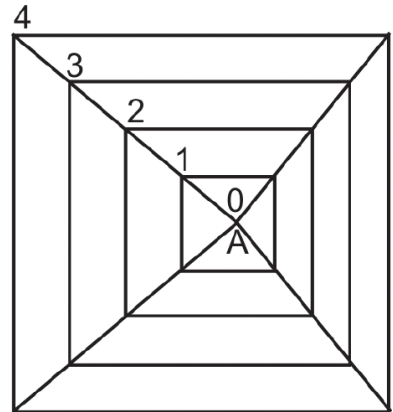

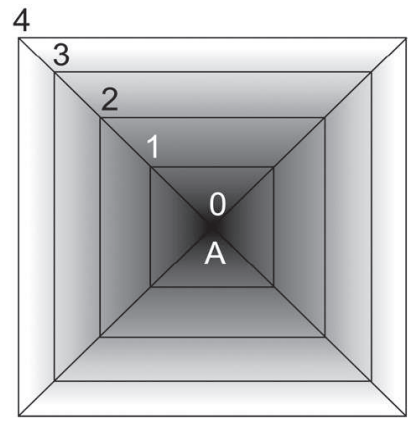

3b Perspectiva

Figura 3. Niveles de profundidad perceptual a intervalos logarítmicos.

El experimento consistió en construir un segundo estereograma (Figura 4) donde los mismos cinco niveles de la pirámide fueron dibujados, ya no a intervalos iguales de paralaje como en la Figura 3, sino con los intervalos que, de acuerdo con la ecuación de Fechner (Ecuación 1), producen intervalos iguales de sensación de profundidad. Como el objetivo 
del experimento no es obtener valores absolutos sino comparar escalas (aritmética y logarítimica), el valor de la constante $K$ puede asignarse arbitrariamente. Además, su valor exacto es aún desconocido. Se tiene entonces que, asignando el valor arbitrario de $K=1$, la Ecuación 1 puede escribirse:

$$
\begin{aligned}
& S=\log E \\
& \therefore E=A n t i \log S
\end{aligned}
$$

Para facilitar su comparación con la Figura 3a, la Figura 4a se dibujó utilizando una pirámide con dimensiones iguales a la de la Figura 3a. El máximo paralaje utilizado en la Figura 3 a fue $8 \mathrm{~mm}$ (cuatro intervalos de $2 \mathrm{~mm}$ cada uno), y este mismo paralaje total fue utilizado para construir el estereograma 4a; o sea que la profundidad perceptual total para ambas pirámides es log8 (Ecuación 2). En razón de que arbitrariamente se tomó $K=1$, los valores de $S$ no pueden darse en unidades específicas, como milímetros o centímetros, sino simplemente como unidades de S. Por razones de simplicidad en los cálculos, y para trabajar con valores logarítimicos positivos, los paralajes se midieron a partir de 10 . En la tabla 1 se indica el procedimiento utilizado para la elaboración del correspondiente estereograma (Figura 4a).

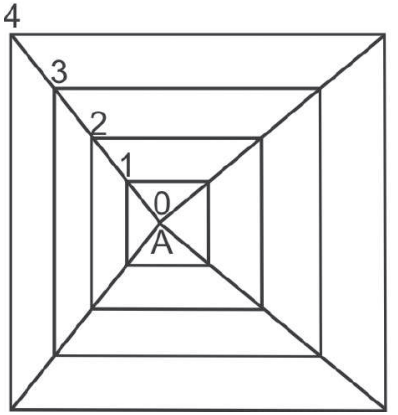

4a Estereograma

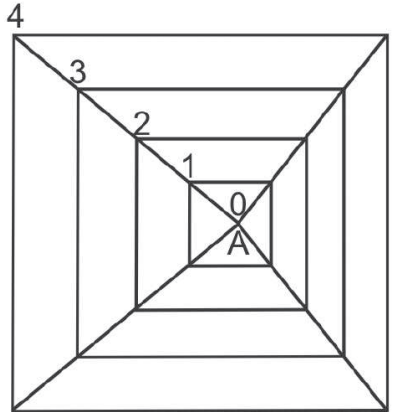

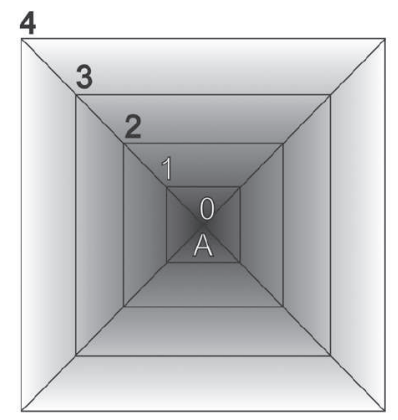

4b Perspectiva

Figura 4. Niveles de profundidad perceptual a intervalos iguales

Tabla 1. Construcción del estereograma de la Figura 4b

\begin{tabular}{|c|r|r|r|r|r|c|}
\hline $\mathbf{A}$ & \multicolumn{1}{|c|}{$\mathbf{B}$} & \multicolumn{1}{c|}{$\mathbf{C}$} & \multicolumn{1}{c|}{$\mathbf{D}$} & $\mathbf{E}$ & $\mathbf{F}$ & $\mathbf{G}$ \\
\hline No. & $\Delta \mathrm{P}_{1} \mathrm{~mm}$. & $\Delta \mathrm{P}+10$ & $\log (\Delta \mathrm{P}+10)$ & $\mathrm{S}$ & Antilog $\mathrm{S}$ & $\Delta \mathrm{P}_{2} \mathrm{~mm}$ \\
\hline 0 & 0 & 10 & 1 & 1 & 10 & 0 \\
\hline 1 & 2 & 12 & 1,079 & 1,064 & 11,59 & 1,59 \\
\hline 2 & 4 & 14 & 1,146 & 1,128 & 13,43 & 3,43 \\
\hline 3 & 6 & 16 & 1,204 & 1,192 & 15,56 & 5,56 \\
\hline 4 & 8 & 18 & 1,255 & 1,256 & 18,03 & 8,03 \\
\hline
\end{tabular}


El contenido de cada una de las columnas de la Tabla 1 se indica a continuación.

Columna A. Número de orden de los niveles de profundidad representados por cuadrados.

Columna B. Muestra la escala de paralaje a intervalos de $2 \mathrm{~mm}$ para cada nivel de la pirámide. Con estos valores se dibujó el estereograma de la Figura $3 a$.

Columna C. Para comodidad en los cálculos, y utilizar valores logarítmicos positivos, se tomó el número 10 como inicio de la escala. Entonces, esta columna muestra valores de paralaje $(10+\Delta \mathrm{P})$ tomados a intervalos de $2 \mathrm{~mm}$., a partir de 10 .

Columna D. Muestra los valores de $\log (10+\Delta P)$. Según la Ecuación 2, estos valores representan las sensaciones de profundidad (S) que serían producidas por los paralajes de la Columna C. En otras palabras, corresponden a las profundidades perceptuales.

Columna E. Resultado de distribuir la profundidad perceptual total de la pirámide $(1,225$ $-1=0,255$ unidades de S) entre 4 intervalos, lo que da un valor aproximado de 0,64 unidades para cada intervalo.

Columna F. Muestra los Valores de antilog S. Son los paralajes que, de acuerdo con la Ecuación 3, producen sensaciones de profundidad a intervalos iguales ( 0,64 cada uno).

Columna $\mathrm{G}$. Indica los valores de paralaje $\Delta \mathrm{P} 2$, resultantes de restar 10 a los paralajes de la Columna F, y así utilizar el origen 0 por conveniencia para el dibujo. Los valores de paralaje mostrados en esta última columna se utilizaron para dibujar el estereograma de la Figura 4. Según la Ecuación de Fechner, tales paralajes, equivalentes a Antilog $S$, deben producir intervalos de profundidad perceptual iguales, como en efecto se observa en el estereograma de la Figura 4.

\section{RESULTADOS Y DISCUSIÓN}

El estereograma de la Figura 3a muestra que las sensaciones de profundidad (S en la ecuación 1) no son proporcionales a los correspondientes paralajes (o estímulos $E$ de la ecuación 1), sino que se ven varíar a intervalos diferentes, aparentemente logarítmicos, de acuerdo con la Ecuación 1. Es decir, intervalos paralácticos iguales no producen intervalos perceptuales iguales.

Por su parte, el estereograma de la Figura 4a indica que intervalos perceptuales iguales se obtienen al utilizar paralajes equivalentes al antilogaritmo de las correspondientes sensaciones de profundidad (antilog S), de acuerdo con la Ecuación 3.

En resumen, los estereogramas de las figuras 3 y 4 indican visualmente que la ley psicofísica de Fechner se cumple para la visión estereoscópica, lo que permite dar un nuevo enfoque al análisis de las relaciones matemáticas que intervienen en el proceso de la visión 
estereoscópica, y por consiguiente hallar nuevos caminos hacia la solución del enigma de la exageración vertical.

Con base en los anteriores resultados, se están realizando pruebas experimentales con un grupo de 20 personas, consistentes en observaciones de gráficos y fotografías estereoscópicas, a fin de determinar la constante $K$ y establecer la ecuación psicofísica que rige la visión estereoscópica.

En principio, la determinación de la constante $K$ puede parecer bastante simple porque se reduciría a determinar experimentalmente valores de las dos variables $(S$ y $E$ ), y con este par de datos calcular $K$. Sin embargo, conviene anotar que, si bien $E$ es susceptible de mediciones físicas, $S$, por el contrario, representa sensaciones mentales difíciles de medir directamente. En este último caso ha sido necesario acudir a métodos indirectos de medición que estamos diseñando con satisfactorios resultados.

\section{CONCLUSIONES}

Se evidencia que en la visión estereoscópica interviene un proceso psicofísico susceptible de formulación matemática. En esa dirección, el próximo paso es determinar la constante $K$ en la ecuación de Fechner, con lo cual se obtendrá una expresión matemática para la percepción tridimensional que permita calcular la exageración vertical.

El desarrollo de tal expresión matemática, objetivo final de la investigación, podría conducir a nuevos desarrollos tecnológicos, particularmente en el campo de la realidad virtual y en la reproducción artificial de determinados efectos visuales. Por ejemplo, haría posible la construcción de instrumentos estereoscópicos con un determinado grado de exageración vertical, o con capacidad para reproducir artificialmente en la mente del observador, un escenario tridimensional con el mismo grado de exageración vertical con que se percibiría en la realidad. Trabajos de investigación actualmente en desarrollo por parte de nuestro grupo de investigación, exploran diversas posibilidades de aplicación.

\section{AGRADECIMIENTOS}

Los autores expresan su reconocimiento al doctor Gottfred Konecny, profesor de la Universidad de Hannover, Alemania, por sus valiosos comentarios sobre los fundamentos científicos de esta investigación. Agradecen al personal de los laboratorios de Ingeniería de la Universidad Militar Nueva Granada (UMNG) su colaboración en el desarrollo de las medidas experimentales. Este trabajo es financiado mediante el proyecto ING-XXXX (2006) de la UMNG. Algunas actividades contaron con el apoyo de Colciencias, a través del programa de movilidad para investigadores. 


\section{REFERENCIAS BIBLIOGRÁFICAS}

[1] ASCHENBRENNER, Claus M., (1952). A Review of Facts and Terms Concerning the Stereoscopic Effect: Photogrammetric Engineering, Vol.18, No 5, pp.818-825.

[2] COLLINS, Stanley H. "Stereoscopic Depth Perception", Photogrammetric Engineering, Vol. 47, No. 1, (1981), 45-52.

[3] FECHNER, G. T., Elemente der psychophysik (Vol. 1) [Elements of psychophysics (Vol. 1)]. Leipzig, Germany: Breitkopf and Härte, (1889).

[4] GOODALE, E.R., "An Equation for Approximating the Vertical Exaggeration of a stereoscopic view", Photogrammetric Engineering, Vol 19, No. 4, (1953), pp.607-616.

[5] LA PRADE, George L, "Stereoscopy - a More General Theory", Photogrammetric Engineering, Vol 38, (1972), No.12.

[6] MILLER, Charles L., "The Stereoscopic Space- Image”, Photogrammetric Engineering, Vol26, No.54, (1958), pp. 810-815.

[7] RAASVELDT, Henri C., The Stereomodel, "How It is Formed and Deformed", Photogrammetric Engineering, Vol. 22. N0. 9, (1956), pp. 708-726.

[8] ROSAS, Humberto, "Vertical Exaggeration in Stereo-Vision", Theories and Facts: Photogrammetric Engineering and Remote Sensing, Vol. 52, No. 11, (1986), 1747-1751.

[9] ROSAS, H., VARGAS, W., CERÓN, A., DOMíNGUEZ, D., CÁRDENAS, A., 2007, "Psychophysical Approach to the Measurement of Depth Perception in Stereo-Vision", $\mathrm{HCl}$ International Conference 2007, Beijing, China, LNCS 4563, Ed Springer.

[10] STONE, Kirk H, 1951. Geographical Air-Photo-Interpretation: Photogrammetric Engineering, Vol. 17, No. 5. Pp. 754-759.

[11] YACOUMELOS, Nick G., "The Geometry of the Stereomodel: Photogrammetric Engineering", Vol. 38, (1972), No. 8.

[12] YACOUMELOS, Nick G., "Comments on Stereoscopy: Photogrammetric Engineering", Vol. 39, No. 3, (1973), p. 274. 
